\title{
Hanle effect from a dipolar magnetic structure: the case of the solar corona and the case of a star
}

\author{
V. Bommier
}

\author{
LESIA, Observatoire de Paris, CNRS-INSU-UMR8109, UPMC Univ. Paris 06, Université Paris Diderot- Paris 7, \\ 5 Place Jules Janssen, 92190 Meudon, France \\ e-mail: v.bommier@obspm.fr
}

Received 11 October 2011 / Accepted 21 January 2012

\section{ABSTRACT}

\begin{abstract}
Context. The context is the magnetic field measurement in external solar or stellar layers by interpreting line polarization measurements and the Hanle effect.

Aims. The aim is to model the Hanle effect depolarization by integrating upon a star on the one hand, and by integrating along a line-of-sight through the solar corona on the other hand.

Methods. The formalism of the atomic density matrix is recalled. Particular attention was devoted to the four axis rotations necessary to transform the magnetic field reference frame into that of the line-of-sight.

Results. In the stellar case, the discrepancy between the results by López Ariste et al. (2011, A\&A, 527, A120) and the symmetry considerations by Ignace etal. (2011, A\&A, 530, A82) is resolved. In the solar case, the computations of the hydrogen Ly $\alpha$ polarization by Derouich et al. (2010, A\&A, 511, A7) are revisited, owing to symmetry considerations.

Conclusions. In the stellar case, we confirm that the effect integrated on a star leads to a non-vanishing magnetic depolarization due to the high non-linearity of the Hanle effect. In the solar case, we find that the Hanle sensitivity of hydrogen $\operatorname{Ly} \beta$ and $\operatorname{Ly} \gamma \operatorname{could}$ be better adapted to the measurement of the coronal background magnetic field. They form a pair of lines of different and complementary sensitivity, which makes it possible to determine the full vector. Ly $\alpha$ would be instead adapted to the coronal loop magnetic field measurement, because this field is stronger and suited to the Ly $\alpha$ Hanle sensitivity.
\end{abstract}

Key words. atomic processes - magnetic fields - polarization - scattering - Sun: corona - stars: magnetic field

\section{Introduction}

The Hanle effect is the modification of the scattering polarization by the magnetic field (Hanle 1924). The scattering polarization is a linear polarization whose direction is perpendicular to the scattering plane, and the Hanle effect is a depolarization and rotation of the polarization direction. More precisely, it is depolarization at right-angle scattering and alternatively hyperpolarization at backward or forward scattering, with a continuous behavior in between. This scattering polarization was observed in solar prominence lines. In addition, the rotation of the polarization direction with respect to the limb was observed. Hyder (1965) reported the first observations of this polarization by Lyot in 1932 and 1935 (Lyot 1934, 1937). Although Öhman (1929) suggested the possibility of the existence of the Hanle effect in off-limb observations, it is Hyder (1965) who ascribed the polarization rotation observed by Lyot to the Hanle effect. Extensive observations were later on undertaken at the Pic-du-Midi with a polarimetric accuracy better by one order of magnitude (Leroy et al. 1977), but without any spectral resolution. Spectrally resolved observations were simultaneously conducted at Sacramento Peak with the experiment Stokes II (Athay et al. 1983). The first interpretation of these observations was incomplete because of the partial anisotropy of the incident radiation of which the integration is difficult (House 1970). The solution was the introduction of the density matrix formalism, where an average atom is embedded in the integrated incident radiation (Bommier 1977; Bommier \& Sahal-Bréchot 1978; Bommier 1980; Landi Degl'Innocenti 1983, 1984). The Hanle effect depends indeed on the so-called "coherences", or interferences between different Zeeman substates, which constitute the "atomic polarization".

The Hanle effect is highly non-linear. It is insensitive to a field aligned with the incident radiation main axis. In right-angle scattering, there is depolarization and rotation of the polarization direction when the field is aligned with the line-of-sight (l.o.s.), but when the field is perpendicular to the scattering plane, there is depolarization but no rotation. This non-linearity led to the idea developed by López Ariste et al. (2011) that the integration on the surface of a star that has a dipolar field would lead to a non-zero global effect. But Ignace et al. (2011) argued that Fig. 3 of López Ariste et al. (2011) does not show the expected symmetry with respect to the central meridian. Besides, Derouich et al. (2010) compute the Hanle effect of the hydrogen Ly $\alpha$ radiation emitted by the solar corona. This requires not a star, but a line-ofsight integration. These authors also assumed a dipolar model for the solar magnetic field. In addition, in a second part they added the effect of a current sheet lying along the whole solar equator, as a very long prominence. For their model without the prominence, their Fig. 5, which displays the polarization rotation as a function of the position angle of the l.o.s. about the solar disk, does not have the required symmetry either. The origin of these discrepancies does probably not lie in the Hanle effect theory itself, which was taken from the completed presentation by Casini (2002) or Landi Degl'Innocenti \& Landolfi (2004), but from the intricate axis rotations from the local magnetic field reference frame having the magnetic field vector as $O z$ axis, to the l.o.s. reference frame all about the star or all along the coronal l.o.s.. Indeed, in principle any reference frame may be transformed into 
any other one by means of a single Euler rotation, but in the practical cases under consideration it is difficult to group in a unique rotation the longitude, latitude, disk center latitude and magnetic field direction that need to be included. Notwithstanding, this can be done by four successive Euler rotations, one for each angle. The formalism of the "irreducible spherical tensors for polarimetry" introduced by Landi Degl'Innocenti (1983) has to be generalized to such a series of linked rotations. To do this, we recall below (Sect. 2) the alternative formalism of the incident and emitted photon density matrices, and we provide the rotation matrix elements. In Sect. 3 we recompute the figures of López Ariste et al. (2011) and we obtain an agreement with Ignace et al. (2011). In Sect. 4 we recompute the Hanle effect of the hydrogen Ly $\alpha$ radiation emitted by the solar corona permeated by a dipolar magnetic field.

\section{Elements of the theory}

The lines studied here are isolated lines with an unpolarizable lower level. We assume that they may be described within the two-level atom aproximation. The lower level is assumed to be $J=0$ or $J=1 / 2$ so that it cannot have any alignment, or $J$ has any value but the lower level alignment is simultaneously destroyed by collisions. Our formalism for computing the Hanle effect is the density matrix formalism as introduced by Bommier \& Sahal-Bréchot (1978) and is well-adapted to the description of a partial polarization. The density matrix was expanded in irreducible tensorial components ${ }^{J J} \rho_{Q}^{K}$ with $K=0,1, \ldots, 2 J$ and $Q=-K,-K+1, \ldots, K-1, K$. We refer to Landi Degl'Innocenti $\&$ Landolfi (2004) for an introduction to irreducible tensorial calculations, in particular p. 127 sqq. for the physical meaning of the multipole moments.

\subsection{Incident photon density matrix}

The incident and reemitted photons are described by their respective density matrices, which are of angular momentum $J=1$ and are in fact generalized polarization matrices. The specific intensity (in $\mathrm{erg} / \mathrm{cm}^{2} / \mathrm{s} / \mathrm{sr} / \mathrm{Hz}$ ) of the radiation incoming at the scattering point $O$ located above the illuminating star, is

$I_{v}=W\left(\frac{2 h v^{3}}{c^{2}}\right) /\left[\exp \left(\frac{h v}{k T_{r}}\right)-1\right]$,

where $T_{r}$ is the radiation temperature of the radiation emitted by the star, and $W$ is the dilution factor, which is the normalized solid angle under which the star is seen from $O$

$W=\frac{\mathrm{d} \Omega}{4 \pi}$.

If the star radiation is limb-darkened following the usual empirical law

$I(i)=1-u+u \cos i$,

where $i$ is the heliocentric angle (the angle between the line-ofsight and the star radius), and $u$ is the limb-darkening parameter, the dilution factor is given by

$$
\begin{aligned}
W= & \frac{1-u}{1-u / 3} \frac{1}{2}(1-\cos \gamma)+\frac{u}{1-u / 3} \\
& \times \frac{1}{4}\left[1-\frac{\cos ^{2} \gamma}{\sin \gamma} \ln \left(\frac{1+\sin \gamma}{\cos \gamma}\right)\right],
\end{aligned}
$$

where $\gamma$ is the half-angle at summit of the cone under which the star is seen from $O$. Following the drawing of Fig. 1 of Sahal-Bréchot (1974), $W$ can be written as the sum of an isotropic part part $W_{\mathrm{i}}$ and an anisotropic part $W_{\mathrm{a}}$ (Charvin 1965). Describing this drawing, the anisotropy of the incident radiation (which is contained inside the cone) is accounted for by the intensity $B$ of the radiation electric field along the local star radius taken as $O z$, lower than the intensity $A$ along the perpendicular axes $O x$ and $O y . W_{\mathrm{i}}$ corresponds to $B$ along each of the three axes, which is an isotropic radiation, whereas $W_{\mathrm{a}}$ corresponds to $A-B$ along $O x$ and $O y$, which is a directive incident radiation along $O z$. The expressions of $W_{\mathrm{i}}$ and $W_{\mathrm{a}}$ are given by van de Hulst (1950) as

$$
\begin{aligned}
W_{\mathrm{i}}= & \frac{1-u}{1-u / 3} \frac{1}{4}(2+\cos \gamma)(1-\cos \gamma)^{2}+\frac{u}{1-u / 3} \\
& \times \frac{3}{32}\left[2+\cos ^{2} \gamma-\frac{\cos ^{2} \gamma\left(4-\cos ^{2} \gamma\right)}{\sin \gamma}\right. \\
& \left.\times \ln \left(\frac{1+\sin \gamma}{\cos \gamma}\right)\right], \\
W_{\mathrm{a}}= & \frac{1-u}{1-u / 3} \frac{1}{4} \cos \gamma\left(1-\cos ^{2} \gamma\right)+\frac{u}{1-u / 3} \\
& \times \frac{1}{32}\left[3 \sin ^{2} \gamma-1+\frac{\cos ^{2} \gamma\left(1+3 \sin ^{2} \gamma\right)}{\sin \gamma}\right. \\
& \left.\times \ln \left(\frac{1+\sin \gamma}{\cos \gamma}\right)\right] .
\end{aligned}
$$

In the reference frame where the quantization axis (the $O z$ axis) lies along the solar radius, the incident photons density matrix elements $\phi_{Q}^{K}$ are given by

$$
\begin{aligned}
\phi_{0}^{0} & =\frac{1}{\sqrt{3}} \\
\phi_{0}^{2} & =\frac{1}{\sqrt{6}} \frac{W_{\mathrm{a}}}{W_{\mathrm{i}}+W_{\mathrm{a}}} \\
\phi_{0}^{1} & =\phi_{ \pm 1}^{1}=\phi_{ \pm 1}^{2}=\phi_{ \pm 2}^{2}=0 .
\end{aligned}
$$

\subsection{Statistical equilibrium of the atomic density matrix}

The case of the $J=0$ (lower level) $\rightarrow J^{\prime}=1$ (upper level) line, usually called the normal Zeeman triplet line, will be considered for the star case, as in López Ariste et al. (2011). For the case of the solar corona, the hydrogen $\operatorname{Ly} \alpha$ line is a $J=1 / 2$ (lower level) $\rightarrow J^{\prime}=3 / 2$ (upper level) line complemented with an unpolarized neighboring line of known intensity $J=1 / 2 \rightarrow J^{\prime}=1 / 2$. Following Bommier \& Sahal-Bréchot (1982), the hyperfine structure can be neglected for computing the scattering polarization of this line. In the following we introduce the statistical equilibrium of the atomic density matrix, embedded in the incoming radiation. The line under consideration comes from a two-level atom, with unpolarized lower level, so that the density matrix has only one element in the lower level $\sqrt{2 J+1} \rho_{0}^{0}=1$. Then, we will denote as $\rho_{Q}^{K}$ the atomic density matrix components in the upper level. Following Bommier (1977) and Bommier \& Sahal-Bréchot (1978), the statistical equilibrium equation for the upper level density matrix element $\rho_{Q}^{K}$ is

$$
\begin{aligned}
\frac{\mathrm{d} \rho_{Q}^{K}}{\mathrm{~d} t}=0= & -\mathrm{i} \omega_{\mathrm{L}} Q \rho_{Q}^{K}-A \rho_{Q}^{K} \\
& +3 B I_{v}(-1)^{1+J+J^{\prime}}\left\{\begin{array}{ccc}
1 & J^{\prime} & J \\
J^{\prime} & 1 & K
\end{array}\right\} \varphi_{Q}^{K},
\end{aligned}
$$


where $A$ and $B$ are the Einstein coefficients of the line under study, $\omega_{\mathrm{L}}$ is the Larmor pulsation

$\omega_{\mathrm{L}}=\frac{g_{J^{\prime}} \mu_{B} B}{\hbar}$,

where $g_{J^{\prime}}$ is the Landé factor of the upper level and $\omega_{\mathrm{L}} / 2 \pi g_{J^{\prime}} B=$ 1.4 MHz/G. $\varphi_{Q}^{K}$ is the incident photon density matrix element, but in the magnetic field reference frame, i.e. the frame where the magnetic field is the $O z$ or quantization axis. Thus, $\varphi_{Q}^{K}$ has to be derived from $\phi_{Q}^{K}$ given above by applying the proper frame rotation matrix. The $6-j$ symbol takes the following value for the lines of interest in the present paper, for $K=0$

$\left\{\begin{array}{ccc}1 & J^{\prime} & J \\ J^{\prime} & 1 & 0\end{array}\right\}=\frac{(-1)^{1+J+J^{\prime}}}{\sqrt{3} \sqrt{2 J^{\prime}+1}}$

and, for $K=2$

$$
\begin{aligned}
& \left\{\begin{array}{lll}
1 & 1 & 0 \\
1 & 1 & 2
\end{array}\right\}=\frac{1}{3} \\
& \left\{\begin{array}{ccc}
1 & 3 / 2 & 1 / 2 \\
3 / 2 & 1 & 2
\end{array}\right\}=-\frac{1}{2 \sqrt{2} \sqrt{3}} .
\end{aligned}
$$

Introducing the $w_{J^{\prime} J}^{(K)}$ coefficient of Landi Degl'Innocenti (1984)

$$
\begin{aligned}
w_{J^{\prime} J}^{(K)} & =\frac{\left\{\begin{array}{ccc}
1 & J^{\prime} & J \\
J^{\prime} & 1 & K
\end{array}\right\}}{\left\{\begin{array}{rrr}
1 & J^{\prime} & J \\
J^{\prime} & 1 & 0
\end{array}\right\}} \\
& =(-1)^{1+J^{+} J^{\prime}} \sqrt{3} \sqrt{2 J^{\prime}+1}\left\{\begin{array}{ccc}
1 & J^{\prime} & J \\
J^{\prime} & 1 & K
\end{array}\right\},
\end{aligned}
$$

one can rewrite the statistical equilibrium equation as

$$
\begin{aligned}
\frac{\mathrm{d} \sqrt{2 J^{\prime}+1} \rho_{Q}^{K}}{\mathrm{~d} t}=0= & -\mathrm{i} \omega_{\mathrm{L}} Q \sqrt{2 J^{\prime}+1} \rho_{Q}^{K} \\
& -A \sqrt{2 J^{\prime}+1} \rho_{Q}^{K} \\
& +B I_{\nu} w_{J^{\prime} J}^{(K)} \sqrt{3} \varphi_{Q}^{K} .
\end{aligned}
$$

The sensitivity of the Hanle effect to the magnetic field strength is governed by the quantity $\omega_{\mathrm{L}} \tau$ (see Eq. (8)), where $\tau$ is the upper-level lifetime $\tau=1 / A$. When $\omega_{\mathrm{L}} \tau \approx 1$ the effect is sensitive to the magnetic field strength that affects the Larmor pulsation $\omega_{\mathrm{L}}$ (see Eq. (9)), whereas when $\omega_{\mathrm{L}} \tau \gg 1$ the effect is saturated with respect to the field strength, and is sensitive to the field direction only. For typical visible lines, $A$ is about $10^{7}$, which leads to a sensitivity to fields of about $10 \mathrm{G}$, which are usually qualified as weak. UV lines will be sensitive to stronger fields because their lifetimes are usually shorter. In terms of field strength, $\omega_{\mathrm{L}} \tau=1$ corresponds to what the authors call the critical, or typical, Hanle field $B_{t}$.

The incident photons density matrix may be simply related to the irreducible tensor $J_{Q}^{K}(v)$ introduced by Landi Degl'Innocenti (1984) for describing the incident radiation effect, by

$\varphi_{Q}^{K}=\frac{1}{I_{v} \sqrt{3}} \int \mathrm{d} v(-1)^{Q} J_{-Q}^{K}(v) \phi\left(v_{0}-v\right)$,

where $\phi\left(v_{0}-v\right)$ is the absorption line profile with the line center frequency $v_{0}$. When the incident radiation is frequency flat, this simply reduces to

$\varphi_{Q}^{K}=\frac{1}{I_{v} \sqrt{3}}(-1)^{Q} J_{-Q}^{K}$.
In the statistical equilibrium equation developed here, we neglected any spectral variation of the incident radiation along the absorption line profile. Raouafi (2002) took into account possible spectral variation of the incident radiation, which acts differently on the different velocity classes of the scatterer. Khan et al. (2011) also addressed this problem for the Ly $\alpha$ line of the solar corona, and Landi Degl'Innocenti (1996) discussed the physical approximations, which are part of the complete redistribution general frame.

\subsection{Re-emitted photon density matrix}

This density matrix is given by

$$
\begin{aligned}
\Phi_{Q}^{K}= & \left(2 J^{\prime}+1\right) A \\
& \times(-1)^{1+J+J^{\prime}+K}\left\{\begin{array}{ccc}
1 & J^{\prime} & J \\
J^{\prime} & 1 & K
\end{array}\right\} \rho_{Q}^{K}
\end{aligned}
$$

in the magnetic field reference frame, where the $O z$ or quantization axis is the field direction. Introducing $w_{J^{\prime} J}^{(K)}$, this emitted photon density matrix can be written as

$$
\sqrt{3} \Phi_{Q}^{K}=A w_{J^{\prime} J}^{(K)}(-1)^{K} \sqrt{2 J^{\prime}+1} \rho_{Q}^{K} .
$$

This frame has to be rotated into the l.o.s. reference frame. This rotation is the object of the following subsection. If we denote as $\Phi_{Q}^{\prime K}$ the density matrix elements in the l.o.s. reference frame, they are related to the Stokes parameters of the radiation by

$$
\begin{aligned}
I & =+\frac{3}{4 \pi}\left(\frac{1}{\sqrt{3}} \Phi_{0}^{\prime 0}+\frac{1}{\sqrt{6}} \Phi_{0}^{\prime 2}\right) \\
Q & =-\frac{3}{4 \pi} \operatorname{Re} \Phi_{2}^{\prime 2} \\
U & =+\frac{3}{4 \pi} \operatorname{Im} \Phi_{2}^{\prime 2} \\
V & =+\frac{3}{8 \pi} \sqrt{2} \Phi_{0}^{\prime \prime}=0 .
\end{aligned}
$$

Strictly speaking, all these constributions have to be multiplied by $n_{\mathrm{a}} h v / 4 \pi$, where $n_{\mathrm{a}}$ is the atom density and $v$ is the line frequency. The positive $Q$ direction (the $O x$ axis of the Stokes parameters reference frame) is the projection of the solar radius onto the plane of the sky. As stated above, the odd $K$ value elements are zero so that there is no circular polarization induced by the Hanle effect (the case of the incomplete Paschen-Back effect is excluded from this discussion).

\subsection{Axis rotations}

The present modeling requires several axis rotations. First, for each scattering point, the incident photon density matrix has to be rotated (strictly speaking, its reference frame) into the magnetic field reference frame. Then the statistical equilibrium can be solved and the re-emitted photon density matrix can follow. This re-emitted photon density matrix has to be rotated (its reference frame) into the l.o.s. reference frame, which can be done by applying several rotations successively, which are: a rotation to go back from the magnetic field reference frame to the solar reference frame ( $O x$ axis along the local parallel), a rotation for the longitude, a rotation for the latitude, a rotation for the inclination of the Sun rotation axis, or disk center latitude $b_{0}$. The density matrix transformation implied by an axis rotation is given by

${ }^{N} \Phi_{Q}^{K}=\sum_{Q^{\prime}} \exp (\mathrm{i} \gamma Q) d_{Q^{\prime} Q}^{K}(\beta) \exp \left(\mathrm{i} \alpha Q^{\prime}\right){ }^{A} \Phi_{Q}^{K}$, 

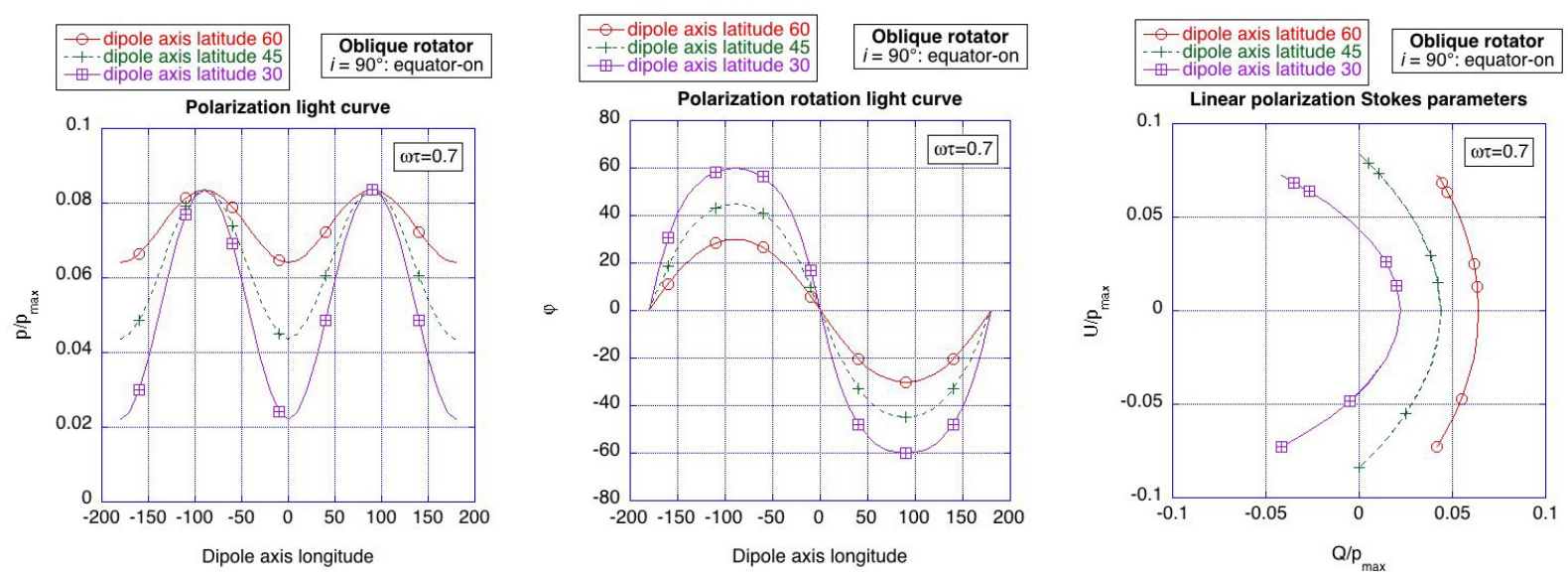

Fig. 1. Stellar case: light curve for the linear polarization degree (left) and direction (middle), and for the Stokes parameters (right) when the magnetic dipole axis is not aligned with the star rotation axis, which is assumed to lie in the plane of the sky. The polarization direction is given by its rotation with respect to the star rotation axis. $p_{\max }$ is the polarization degree that would be observed at a star limb point in zero magnetic field. This figure replaces Fig. 3 of López Ariste et al. (2011).
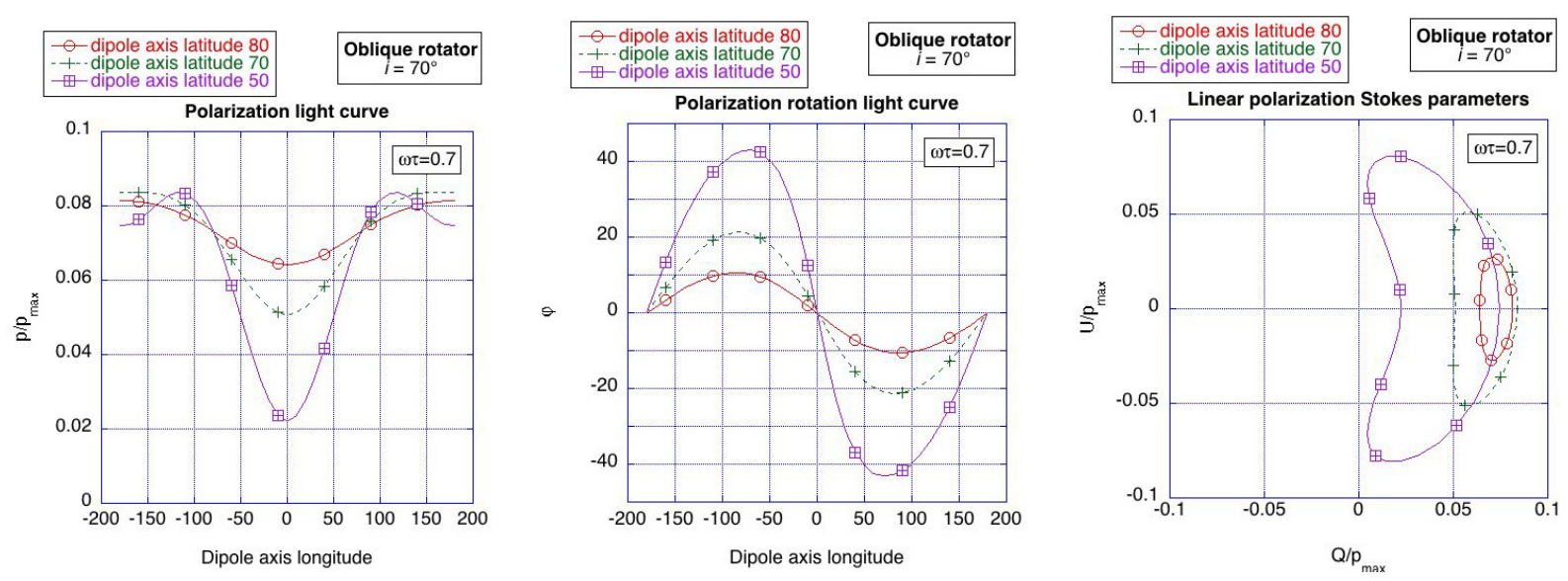

Fig. 2. Same as Fig. 1, but for the case of a star rotation axis inclined with respect to the line-of-sight.

where ${ }^{N} \Phi_{Q}^{K}$ is the density matrix element in the new reference frame, ${ }^{A} \Phi_{Q}^{K}$ is the density matrix element in the old reference frame, $(\alpha, \widetilde{\beta}, \gamma)$ are the Euler angles of the rotation that ransforms the old reference frame into the new one. A rotation of Euler angles $(\alpha, \beta, \gamma)$ is achieved by applying successfully 1$)$ a rotation of angle $\alpha$ about the $O z$ axis of the old reference frame; this rotation brings the $O y$ axis of the old frame into the $O u$ position; 2) a rotation of angle $\beta$ about $O u$, which brings $O z$ into the $O Z$ axis of the new reference frame; 3 ) a rotation of angle $\gamma$ about $O Z$ to bring $O u$ into the $O Y$ axis of the new reference frame. The rotations matrix elements $d_{Q^{\prime} Q}^{K}(\beta)$ are tabulated in Brink \& Satchler (1994).

\section{Case of a star with a dipolar magnetic field}

In a recent paper, López Ariste et al. (2011) pointed out that, owing to its non-linearity, the Hanle effect operating on a dipolar magnetic field should result in a non-zero linear polarization of the global radiation emitted by the star. Roughly speaking, a dipolar field is nearly vertical near the poles, whereas it is horizontal at the equator, in the star reference frame. A vertical field, which is aligned with the anisotropy direction of the incident radiation, does not show any Hanle effect or depolarization. In contrast, the horizontal field is responsible for a depolarization of the scattered radiation by the Hanle effect (but no rotation of the polarization direction). Therefore a global linear polarization remains after integration on the whole star, because the equatorial radiation is more depolarized than the polar one, with no rotation of the polarization direction.

However, Ignace et al. (2011) remarked that Fig. 3 of López Ariste et al. (2011), which is the star polarization light curve when the magnetic dipole axis is not aligned with the star rotation axis, does not display the expected symmetry with respect to the star central meridian. But Ignace et al. (2011) did not any calculation. We did the calculations and obtained the results presented in Fig. 1. We find a behavior of the polarization light curve very different from Fig. 3 of López Ariste et al. (2011). Our plot clearly displays the symmetry required by Ignace et al. (2011). This curve was plotted for the "equator-on" geometry, where the star rotation axis lies in the plane of the sky. For generalization, we considered the case of a star rotation axis inclined with respect to the plane of the sky, which we plotted in Fig. 2. The expected symmetry is also present.

In addition, we replotted Fig. 1 of López Ariste et al. (2011), which we also found to differ and reproduce it in Fig. 3. This figure represents the dependence of the star linear polarization, scaled to the zero-field polarization per limb point as a function of the magnetic field strength scaled as $\omega_{\mathrm{L}} \tau$. We show that the linear polarization degree reaches a maximum at about $\omega_{\mathrm{L}} \tau=0.8$ and then decreases. This decrease was not obtained 


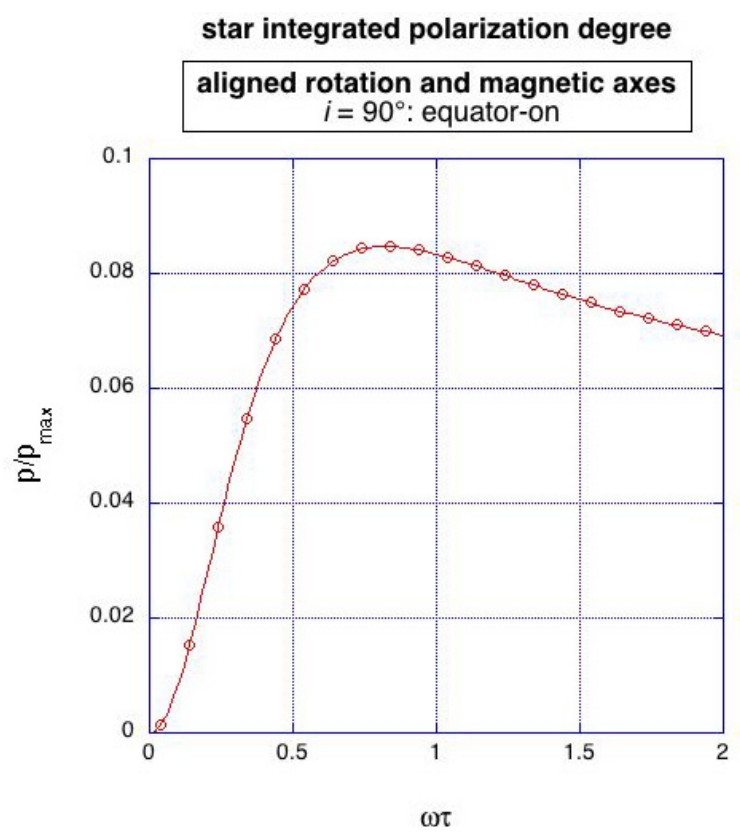

Fig. 3. Stellar case: linear polarization degree of the star radiation as a function of the magnetic field strength expressed in the reduced form where $\omega$ is the Larmor pulsation and $\tau$ is the upper level lifetime. $p_{\max }$ is the polarization degree that would be observed at a star limb point in a zero magnetic field. This figure replaces Fig. 1 of López Ariste et al. (2011).

by López Ariste et al. (2011) and results from the progressive saturation of the Hanle effect.

The dipolar magnetic field is given by

$B=B_{0}\left(\frac{R_{*}}{R}\right)^{3}[3(\hat{M} \cdot \hat{R}) \hat{R}-\hat{M}]$,

where the hat denotes the vector direction, or unit vector. $\boldsymbol{R}$ is the local star radius and $\boldsymbol{M}$ is the dipolar moment. $B_{0}$ is the magnetic field strength at the star surface and at the magnetic equator. We modeled the star emission as that of a half-sphere of radius $R_{*}$. We assumed a normal Zeeman triplet line $J=0$ (lower) $\rightarrow J^{\prime}=1$ (upper) $\left(w_{J^{\prime} J}^{(2)}=1\right)$, and for the calculation we considered a directive incident radiation along the stellar radius $\left(W_{\mathrm{i}}=0, W_{\mathrm{a}}=1\right)$. In this very theoretical case, the zero-field polarization degree per limb point is unity. In practice this $p_{\max }$ is not unity, owing to partially directive incident radiation, but the ratio $p / p_{\max }$ remains unchanged. The case of another line could be considered by varying $w_{J^{\prime} J}^{(2)}$, which also changes $p_{\max }$ but not $p / p_{\max }$. This description of the formation of the polarization of the star radiation lies in the frame of the last scattering approximation, where the polarization is formed in the last scattering before the photon is emitted from the star. Owing to the weakness of the polarization degree, this approximation is quite valid. Its validity has been extensively studied by Sampoorna et al. (2009).

\section{Case of the hydrogen Ly $\alpha$ line of the solar corona permeated by a dipolar magnetic field}

Similarly, we observed that Fig. 5 of Derouich et al. (2010) does not display the expected symmetry, so that we also recomputed the calculations. These authors were modeling the Hanle effect of the hydrogen Ly $\alpha$ line emitted in the solar corona by scattering of the chromosphere-corona transition zone radiation. The corona magnetic field was simply modeled by a dipole, as in the previous section. The authors considered that the dipole axis and the sun rotation axis were aligned, and were both in the plane of the sky. We considered the same. In the following we evaluate the polarization observed along a line-of-sight intersecting the plane of the sky in $P$, which is at the position angle $\varphi$ with respect to the solar north pole (see Fig. 3 of Derouich et al. 2010). Figure 5 of Derouich et al. (2010) displays the rotation of the polarization direction, with respect to the zero-field case, as a function of $\varphi$. Indeed, when the star is spatially resolved, as in this Section, the zero-field polarization direction is the limb direction, and the polarization direction of the scattered radiation is rotated by the Hanle effect. The dipolar magnetic field is symmetrical with respect to the equator plane, in the pseudo-vector symmetry. Consequently the emitted polarization must also be symmetrical with respect to this plane, which means that when $\varphi$ is changed to $180^{\circ}-\varphi$, the polarization direction is the symmetrical one and the rotation of the polarization direction is changed to its opposite. As a consequence, the rotation of the polarization direction at the equator $\varphi=90^{\circ}$, must be zero. This is not the case of Fig. 5 of Derouich et al. (2010). We performed the calculation and obtained instead Fig. 4 (lower part), which obeys the symmetry. The upper part of Fig. 4 displays the dependence of the linear polarization degree, and is also different from the corresponding Fig. 4 of Derouich et al. (2010). In particular, in contrast to these authors, our polarization degree remains always lower than the zero-field one, as expected for Hanle effect in such a scattering.

In the following, Derouich et al. (2010) added to their model the effect of a current sheet lying along the whole solar equator, as a very long prominence. Their Figs. 4 and 5, to which we compare our results were obtained in the purely dipolar field model, without the current sheet. We remark that, following their Fig. 7, the effect of the current sheet is of the first order. We also remark that the addition of the current sheet all along the equator does not break the symmetry of the field with respect to the equator, so that the rotation of the polarization direction at the equator $\varphi=90^{\circ}$ must remain zero.

The hyperfine structure is negligible for computing the hydrogen Ly $\alpha$ scattering polarization (Bommier \& Sahal-Bréchot 1982), because the hyperfine splitting is comparable to, or smaller than, the natural width. The hydrogen Ly $\alpha$ line is $\mathrm{aD}_{1}-\mathrm{D}_{2}$ lines system formed by Ly $\alpha_{1}$ that is $J=1 / 2$ (lower) $\rightarrow$ $J^{\prime}=1 / 2$ (upper), and $\operatorname{Ly} \alpha_{2}$ that is $J=1 / 2$ (lower) $\rightarrow J^{\prime}=3 / 2$ (upper). $\operatorname{Ly} \alpha_{1}$ is unpolarizable because of $J^{\prime}=1 / 2$. The statistical equilibrium can be solved independently for the two lines. The Einstein spontaneous emission coefficient $A$ is the same for $\operatorname{Ly} \alpha_{1}$ and $\operatorname{Ly} \alpha_{2}$, and the Einstein absorption coefficient $B$ is twice weaker for $\operatorname{Ly} \alpha_{1}$ than for $\operatorname{Ly} \alpha_{2}$. For the emitted photon density matrix, $\operatorname{Ly} \alpha_{1}$ has $\Phi_{0}^{0}$ half of that of $\operatorname{Ly} \alpha_{2}$ and no other $\Phi$ s, so that Ly $\alpha_{1}$ is simply taken into account by computing Ly $\alpha_{2}$ following the merthod described above, and mutliplying the derived $\Phi_{0}^{0}$ by 1.5 .

The magnetic field was assumed to be that of a dipole (see Eq. (20)). The radiation was integrated along the line-of-sight, assuming an optically thin $\operatorname{Ly} \alpha$ emission. The coronal hydrogen density was scaled from the electron density given by Allen (1973) with the values given in Fig. 2 caption of Cranmer (1998). We give here the results for the corona of solar maximum, but the case of the corona of solar minimum (different in pole and equator) is not very different. We applied $B_{0}=80 \mathrm{G}$ for the equator solar surface magnetic field to obtain the figures closest to (though different) Derouich et al. (2010), who claimed that they took " 15 to $20 \mathrm{G}$ close to the base of the corona". We do not 

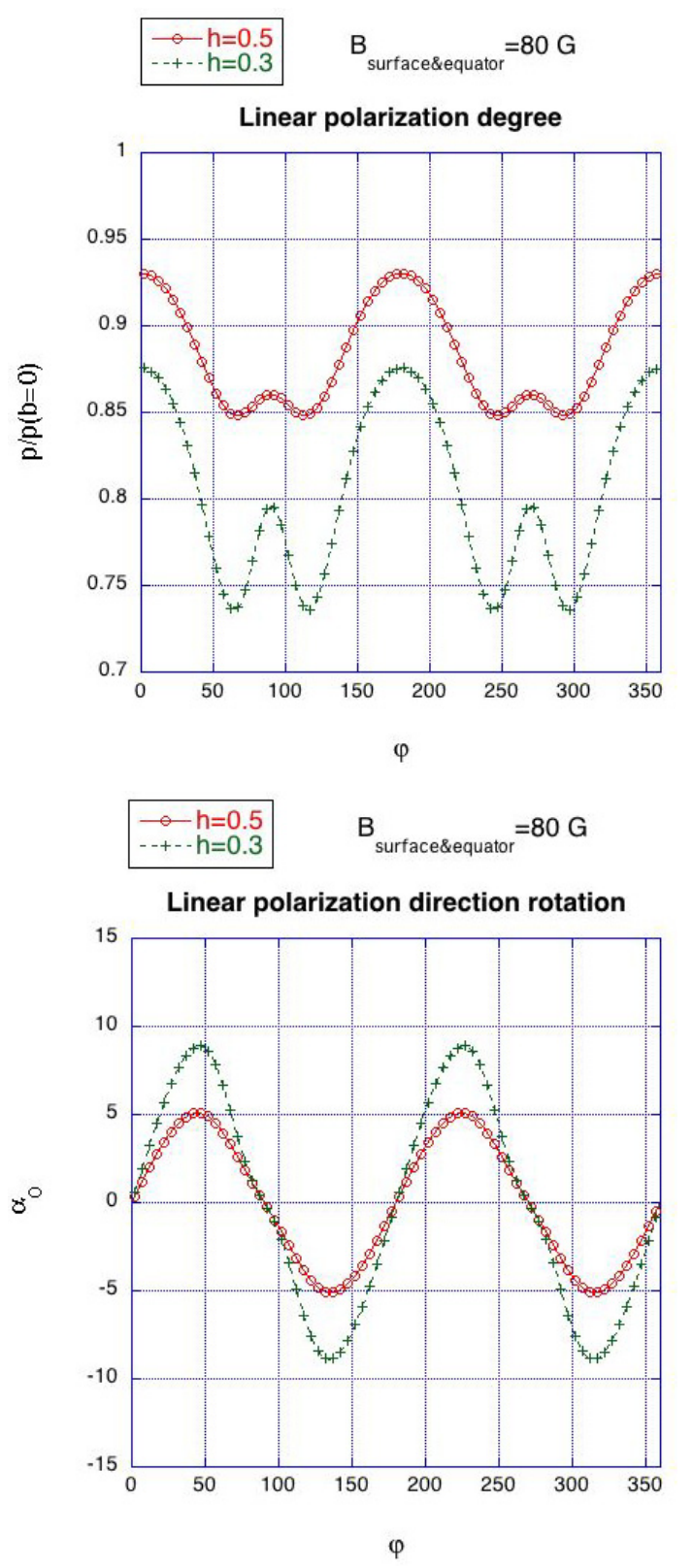

Fig. 4. Solar case: Ly $\alpha$ linear polarization degree (top) and direction (bottom) for two apparent heights above the solar limb (in solar radius), as a function of the position angle of the line-of-sight with respect to the sun rotation axis. This figure replaces Figs. 4 and 5 of Derouich et al. (2010).

understand this difference, and our value of $80 \mathrm{G}$ is consistent with the Hanle sensitivity of the $\operatorname{Ly} \alpha$ line as reported in Table 1, considering in addition that the dipole magnetic field decreases as $\left(R_{\odot} / R\right)^{3}$, so that with $80 \mathrm{G}$ at the solar surface one has $36 \mathrm{G}$ at $0.3 R_{\odot}$ above the surface. In this table, it can be seen that the Hanle sensitivity of $\operatorname{Ly} \beta$ or $\operatorname{Ly} \gamma$ could be better adapted to what is expected for the coronal magnetic field. The dependance of their polarization can be derived from the Ly $\alpha$ one by scaling with the respective Hanle sensitivity.

\section{Conclusion}

We aimed to study the Hanle effect in a dipolar magnetic field, stellar or solar. As suggested by López Ariste et al. (2011), we found that the effect integrated on a star leads to a non-vanishing

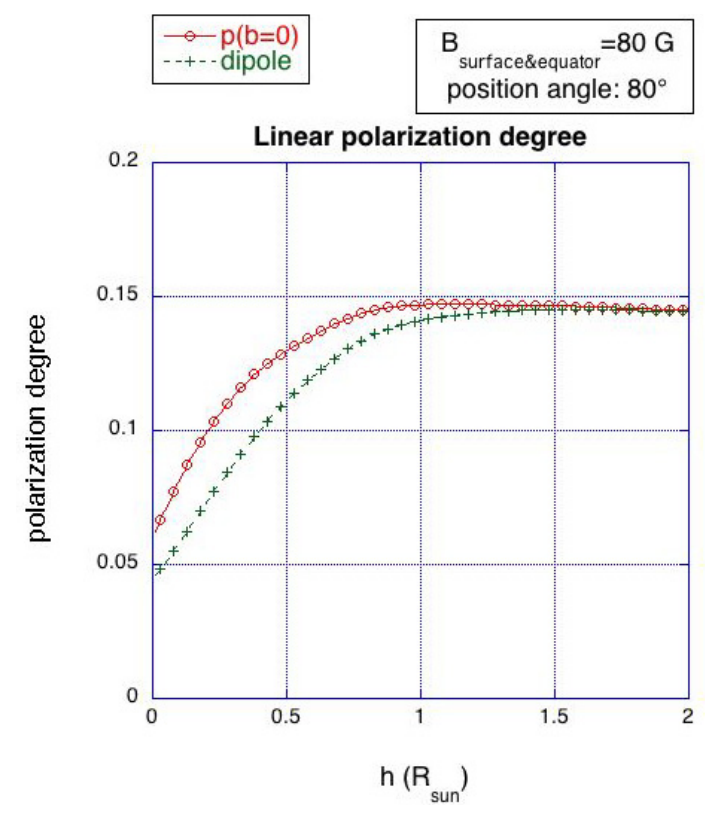

Fig. 5. Solar case: Ly $\alpha$ linear polarization as a function of the apparent height of the line-of-sight above the solar limb, at the position angle $80^{\circ}$ with respect to the solar rotation axis. Circles: case of the zero magnetic field. Crosses: case of the dipolar magnetic field (see text). This figure replaces Fig. 7 of Derouich et al. (2010), except for the case of the additional equatorial current sheet, which we did not compute.

Table 1. Hanle sensitivity (in G) of the first lines of the hydrogen Lyman series.

\begin{tabular}{ccc}
\hline \hline Line & $\begin{array}{c}\text { Wavelength } \\
\AA\end{array}$ & $\begin{array}{c}B_{\mathrm{t}} \\
\mathrm{G}\end{array}$ \\
\hline $\mathrm{Ly} \alpha$ & 1216 & 53.2 \\
$\mathrm{Ly} \beta$ & 1026 & 16 \\
$\mathrm{Ly} \gamma$ & 992 & 7 \\
\hline
\end{tabular}

Notes. This sensitivity is defined as the field strength $B_{\mathrm{t}}$ (typical field) for which $\omega \tau=1$, where $\omega$ is the Larmor pulsation and $\tau$ is the upper level lifetime. All the transitions leaving the upper level are taken into account in the lifetime. From Sahal-Bréchot (1981, Table I).

magnetic depolarization due to the high non-linearity of the Hanle effect, which could be of interest for stellar magnetic field diagnostic.

In the solar case, we considered the Hanle effect of the first lines of the Lyman series of hydrogen lines, emitted by the optically thin solar corona permeated by a dipolar magnetic field. These lines have decreasing and complementary Hanle sensitivities, with typical field strengths of 53.2, 16 and $7 \mathrm{G}$, respectively, starting from $\operatorname{Ly} \alpha$ (see Table 1). As for the Hanle effect of solar prominences (Bommier et al. 1981), two lines of different sensitivity are necessary to recover the three field vector coordinates. Ly $\beta(1026 \AA)$ and Ly $\gamma(992 \AA)$ are good candidates to be these two lines for the background coronal field, which is expected to be on the order of a few G. Alternatively, the full vector can be recovered from one line measurement plus one information. Therefore, $\operatorname{Ly} \alpha(1216 \AA)$ is a good candidate for diagnosing the coronal loop magnetic field, which is stronger (hundred $G$ are expected), because the loop shape provides the supplementary information about the field direction, if one assumes that the field lies along the loop. The loop shape delineates the projection of the field direction onto the plane of the sky (see Bommier et al. 1981, for the feasibility of the inversion method). Because 
the coronal magnetic field is very poorly known, such measurements would be welcome and were already proposed for several spatial missions (see for instance Peter et al. 2012).

\section{References}

Allen, C. W. 1973, Astrophysical Quantities, third edn. (The Athlone Press, University of London)

Athay, R. G., Querfeld, C. W., Smartt, R. N., Landi Degl'Innocenti, E., \& Bommier, V. 1983, Sol. Phys., 89, 3

Bommier, V. 1977, Ph.D. Thesis, Université Pierre et Marie Curie Paris 6, Thèse de 3ème cycle

Bommier, V. 1980, A\&A, 87, 109

Bommier, V., \& Sahal-Bréchot, S. 1978, A\&A, 69, 57

Bommier, V., \& Sahal-Bréchot, S. 1982, Sol. Phys., 78, 157

Bommier, V., Sahal-Bréchot, S., \& Leroy, J. L. 1981, A\&A, 100, 231

Brink, D. M., \& Satchler, G. R. 1994, Angular Momentum, third edn. (Oxford: Clarendon Press)

Casini, R. 2002, ApJ, 568, 1056

Charvin, P. 1965, Ann. Astrophys., 28, 877

Cranmer, S. R. 1998, ApJ, 508, 925

Derouich, M., Auchère, F., Vial, J. C., \& Zhang, M. 2010, A\&A, 511, A7

Hanle, W. 1924, Z. Phys., 30, 93
House, L. L. 1970, J. Quant. Spec. Radiat. Transf., 10, 1171

Hyder, C. L. 1965, ApJ, 141, 1374

Ignace, R., Hole, K. T., Cassinelli, J. P., \& Henson, G. D. 2011, A\&A, 530, A82

Khan, A., Belluzzi, L., Landi Degl'Innocenti, E., Fineschi, S., \& Romoli, M. 2011, A\&A, 529, A12

Landi Degl'Innocenti, E. 1983, Sol. Phys., 85, 3

Landi Degl'Innocenti, E. 1984, Sol. Phys., 91, 1

Landi Degl'Innocenti, E. 1996, Sol. Phys., 164, 21

Landi Degl'Innocenti, E., \& Landolfi, M. 2004, Astrophysics and Space Science Library, Vol. 307, Polarization in Spectral Lines (Kluwer Academic Publishers)

Leroy, J. L., Ratier, G., \& Bommier, V. 1977, A\&A, 54, 811

López Ariste, A., Asensio Ramos, A., \& González Fernández, C. 2011, A\&A, 527, A 120

Lyot, B. 1934, Compte-rendus des séances de l'Académie des sciences, 198, 250

Lyot, B. 1937, L'Astronomie, 51, 203

Öhman, Y. 1929, MNRAS, 89, 479

Peter, H., Abbo, L., Andretta, V., et al. 2012, Experimental Astronomy, in press [arXiv: 1108.5304]

Raouafi, N.-E. 2002, A\&A, 386, 721

Sahal-Bréchot, S. 1974, A\&A, 36, 355

Sahal-Bréchot, S. 1981, Space Sci. Rev., 29, 391

Sampoorna, M., Stenflo, J. O., Nagendra, K. N., et al. 2009, ApJ, 699, 1650

van de Hulst, H. C. 1950, Bull. Astron. Inst. Netherlands, 11, 135 